\title{
Second-Order Effects in Fiber Optical Parametric Amplifier
}

\author{
Kenneth K. Y. Wong \\ Photonic Systems Research Laboratory, Department of Electrical and Electronic Engineering, \\ The University of Hong Kong, Pokfulam, Hong Kong \\ E-mail:kywong@eee.hku.hk
}

\begin{abstract}
Until recently, impressive performance of fiber optical parametric amplifiers (OPAs) has been demonstrated in different respects. However, second-order effects should be addressed before OPAs can be practical. Here we report some of these effects, either exploiting them as in the parametric processor or suppressing them as in the optical amplifier.

(C)2009 Optical Society of America

OCIS codes: (190.4410) Nonlinear optics, parametric processes; (060.2330) Fiber optics communications.
\end{abstract}

\section{Introduction}

In recent years, impressive performance has been demonstrated by the author's research group and different researchers in fiber OPAs in several respects: 1) Gain in excess of 60-dB has been obtained [1]; 2) Fiber OPAs can exhibit a large variety of gain spectra: a gain bandwidth of $400-\mathrm{nm}$ has been demonstrated; tunable narrowband gain regions can also be generated [2]; 3) Noise figure of 3.7- $\mathrm{dB}$ [3], limited by other third-order nonlinear process [4]; 4) Polarization-insensitive operation in both one-pump and two-pump configurations [5]. Besides using fiber OPA in continuous-wave regime as in typical systems, pulsed-pump has also been demonstrated to achieve larger bandwidth and higher peak gain by combining with optical filtering technique [6]. Furthermore, by modulating the pump it is possible to modulate signal and/or idler at the output. This can be used to implement a variety of signal processing functions, including: demultiplexing of optical time-division-multiplexed signals [7]; optical sampling [8], etc.

However, second-order effects should be addressed in order for fiber OPAs to be useful in communication applications. In multi-wavelength systems, these are: four-wave mixing (FWM), cross-phase modulation (XPM); and cross-gain modulation (XGM) between signals [9], especially the signal crosstalk due to the FWM and XGM effects in WDM systems. On the other hand, one can leverage on the peculiar performance of fiber OPAs to achieve some versatile optical signal processing functionalities with appropriate designs.

\section{Suppression of second-order effects}

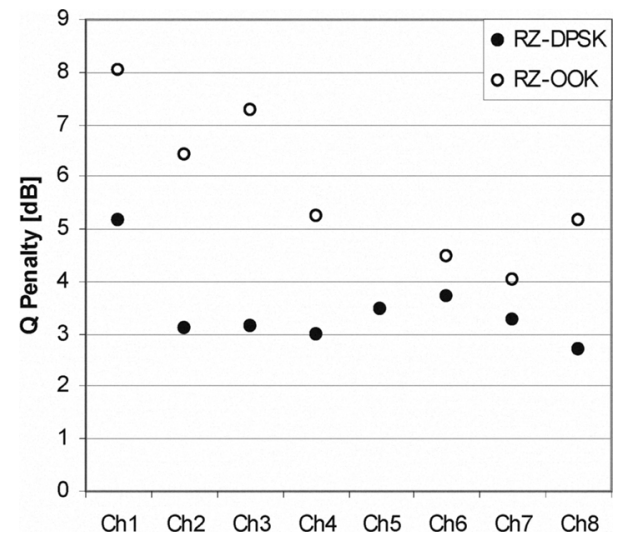

Fig. 1. Q-factor penalty of RZ-DPSK and RZ-OOK signals at different channel [12]

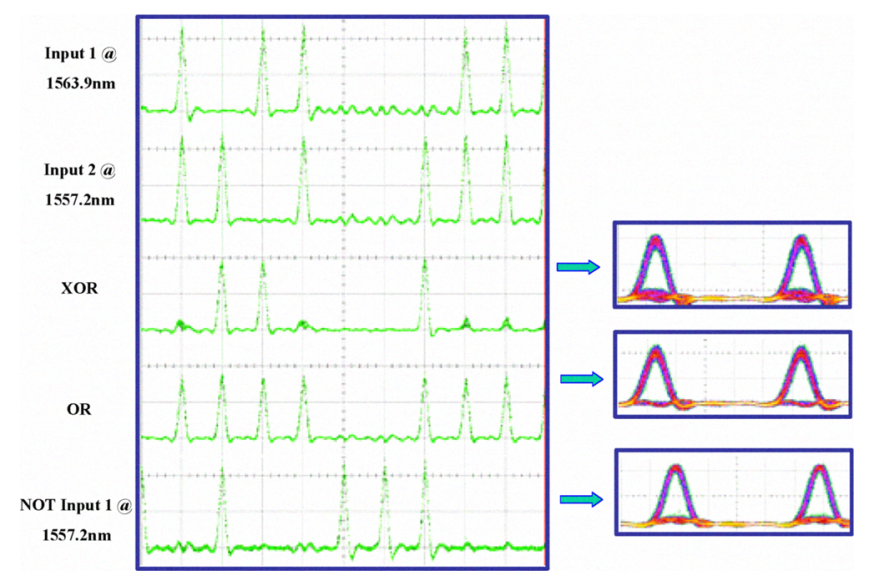

Fig. 2. Bit patterns for the inputs and outputs of XOR-, OR-, and NOT-gates [13].

The author's research group has proposed different schemes to suppress such kind of crosstalk. Namely, the twoorthogonal pumps OPA (2OP-OPA) [10]; WDM signals with polarization-interleaving (POIN) [11]; and recently by return-to-zero differential phase-shift-keying (RZ-DPSK) modulation signal format which suppress the power penalty due to crosstalk, by comparing the eye-diagrams and bit-error-rate (BER) of different formats [12]. We demonstrated substantial crosstalk suppression in one-pump OPA by using RZ-DPSK modulation format, of which its pattern independent amplitude and sub-unity duty cycle would be effective in reducing XGM and FWM effects significantly. By using RZ-DPSK format with eight $10 \mathrm{~Gb} / \mathrm{s}$ channel, $100 \mathrm{GHz}$ channel spacing, Q-factor penalty of RZ-DPSK signal was reduced by $2.4 \mathrm{~dB}$ (on average) comparing to RZ-OOK counterparts as shown in Fig. 1. 


\section{Utilization of second-order effects}

Turns out some of the detrimental second-order effects in fiber OPA are like the double-edge sword, which can be utilized for optical signal processing functionalities that are not available by conventional techniques. Some of the research efforts pursued by our research group will be described here as follows.

Firstly, all-optical XOR-, OR-, NOT-, and AND-gate were demonstrated for $10 \mathrm{~Gb} / \mathrm{s}$ RZ-OOK signals using a single experimental setup. Typical results are shown in Fig. 2. The operating principles of these logic gates based on pump depletion (XGM) and FWM. This setup can be set to generate different logic by altering input power, state-ofpolarization, center frequencies of input optical signals, and/or input bitstreams. Full-width at half-maximum (FWHM) pulsewidth of the input and the output signals were less than $5.1 \mathrm{ps}$ in all cases showing a possibility of higher data rate operation at $80 \mathrm{~Gb} / \mathrm{s}$ or beyond [13].

In addition, we proposed another scheme, using a two-pump OPA (2P-OPA), to achieve both inverted and noninverted all-optical wavelength conversion [14]. The operating principle is again based on the utilization of the XGM of the pump as before. Experiments have confirmed that the signal at $1554 \mathrm{~nm}$ could be flexibly converted to the C-band (1532-1556 nm) with an extinction ratio (ER) between 7 and $14 \mathrm{~dB}$. Noted that the similar scheme can be extended to all-optical wavelength conversion and multicasting for a $10 \mathrm{~Gb} / \mathrm{s} \mathrm{NRZ}$ system with $100 \mathrm{GHz}$ channel spacing. Multicasting is another potentially useful networking function. It involves routing the same data stream from a single node to several destinations, commonly implemented via IP digital routers in electrical domain [15].

\section{Conclusions}

Substantial progress has been made in recent years with the development of fiber OPAs. However, second-order effects should be addressed before OPAs can be practical. Here we reported some of these effects, either exploiting them as in the parametric processor or suppressing them as in the wideband optical amplifier. We anticipate that further progress with high-power pumps, highly-nonlinear fibers with tailored dispersion properties, and SBS suppression techniques, fiber OPAs and related devices will find practical applications in areas such as high-power wavelength conversion, optical communications, optical signal processing, etc.

\section{Acknowledgment}

The work described in this paper was partially supported by grants from the research Grants Council of the Hong Kong Special Administrative Region, China (Project No. HKU 7172/07E and HKU 7179/08E).

\section{References}

[1] T. Torounidis, P. A. Andrekson, and B. E. Olsson, "Fiber-optical parametric amplifier with 70-dB gain," PTL 18, 1194-1196 (2006).

[2] M. E. Marhic, K. K. Y. Wong, and L. G. Kazovsky, "Wideband tuning of the gain spectra of one-pump fiber optical parametric amplifiers," JSTQE 10, 1133-1141 (2004).

[3] J. L. Blows and S. E. French, "Low-noise-figure optical parametric amplifier with a continuous-wave frequency-modulated pump," OL 27, 491-493 (2002)

[4] P. L. Voss and P. Kumar, "Raman-noise-induced noise-figure limit for $\chi^{(3)}$ parametric amplifiers," OL 29, 445-446 (2004).

[5] K. K. Y. Wong, M. E. Marhic, K. Uesaka, and L. G. Kazovsky, "Polarization-independent two-pump fiber optical parametric amplifier," PTL 14, 911-913 (2002).

[6] G. Kalogerakis, K. Shimizu, M. E. Marhic, K. K. Y. Wong, K. Uesaka, and L. G. Kazovsky, "High repetition-rate pulsed-pump fiber optical parametric amplifier for amplifications of communication signals,"JLT 24, 3021-3027 (2006).

[7] J. Hansryd and P. A. Andrekson, "O-TDM demultiplexer with 40-dB gain based on a fiber optical parametric amplifier," PTL 13, 732-734 (2001).

[8] J. Li, J. Hansryd, P. O. Hedekvist, P. A. Andrekson, and S. N. Knudsen, “300-Gb/s eye-diagram measurement by optical sampling using fiber-based parametric amplification," PTL 13, 987-989 (2001).

[9] T. Torounidis, H. Sunnerud, P. Hedekvist and P. A. Andrekson, "Amplification of WDM signals in fiber-based optical parametric amplifiers," PTL 15, 1061-1063 (2003).

[10] K. K. Y. Wong, G.-W. Lui, and L.-K. Chen, "Experimental study of the WDM signal crosstalk in two-pump fiber optical parametric amplifiers," Optics Comm. 270, 429-432 (2007).

[11] K. K. Y. Wong, G. W. Lu and L. K. Chen, "Polarization-interleaved WDM signals in a fiber optical parametric amplifier with orthogonal pumps," OE 15, 56-61 (2007).

[12] B. P. P. Kuo, P. C. Chui, and K. K. Y. Wong, "A Comprehensive Study on Crosstalk Suppression Techniques in Fiber Optical Parametric Amplifier by Modulation Format," JSTQE 14, 659-665 (2008).

[13] D. M. F. Lai, C. H. Kwok, and K. K. Y. Wong, "All-Optical Picoseconds Logic Gates based on a Fiber Optical Parametric Amplifier," OE 16, 18362-18370 (2008)

[14] K. K. Y. Wong, G.-W. Lui, and L.-K. Chen, "Simultaneous all-optical inverted and non-inverted wavelength conversion using a singlestage fiber optical parametric amplifier," PTL 18, 1442-1444 (2006).

[15] K. K. Y. Wong, G.-W. Lui, K.-C. Lau, P. K. A. Wai, and L.-K. Chen, "All-Optical Wavelength Conversion and Multicasting by Cross-Gain Modulation in a Single-Stage Fiber Optical Parametric Amplifier,” OFC2007. 